\title{
Propiedades Físicas del Jugo de Uchuva (Physalis peruviana) Clarificado en Función de la Concentración y la Temperatura
}

\author{
Gloria I. Giraldo(1), Cristian D. Cruz ${ }^{(2)}$ y Nancy R. Sanabria(2)* \\ Universidad Nacional de Colombia, (1) Departamento de Física y Química, Facultad de Ciencias Exactas y \\ Naturales, (2) Departamento de Ingeniería Química, Facultad de Ingeniería y Arquitectura, Campus la \\ Nubia Km 4 Vía al Magdalena, AA 127 Manizales, Colombia. (e-mail: gigiraldogo@unal.edu.co; \\ cdcruzr@unal.edu.co; nrsanabriago@unal.edu.co)
}

* Autor a quien debe ser dirigida la correspondencia

Recibido Jun. 22, 2016; Aceptado Ago. 31, 2016; Versión final Oct. 19, 2016, Publicado Feb. 2017

\section{Resumen}

Se estudió el efecto de la concentración (20 a $50^{\circ}$ Brix) y la temperatura $\left(20\right.$ a $\left.50{ }^{\circ} \mathrm{C}\right)$ sobre la densidad y viscosidad del jugo de uchuva (Physalis peruviana) clarificado, empleando un diseño aleatorio con arreglo de dos factores: temperatura con cinco niveles, concentración con siete niveles y tres repeticiones. También se determinó el punto de congelación del jugo de uchuva en función de la concentración. Los valores de densidad y viscosidad del jugo disminuyeron al aumentar temperatura y se incrementaron al aumentar la concentración de sólidos solubles. El efecto de la temperatura sobre la viscosidad del jugo a diferentes concentraciones fue descrito por una relación tipo Arrhenius, encontrándose una energía de activación de flujo en el rango de 7.02 a $17.62 \mathrm{~kJ} / \mathrm{mol}$. Los puntos de congelación del jugo se calcularon a partir de las curvas de enfriamiento y los resultados se compararon con los estimados a partir de un modelo aplicable a jugos de frutas, con un error relativo promedio del $6.6 \%$.

\section{Physical Properties of Clarified Cape Gooseberry (Physalis peruviana) Juice as a Function of Concentration and Temperature}

\begin{abstract}
The effect of concentration (20 to $50{ }^{\circ}$ Brix) and temperature $\left(20\right.$ to $50{ }^{\circ} \mathrm{C}$ ) on density and viscosity of clarified cape gooseberry (Physalis peruviana) juice was studied, using a randomized arrangement of two factors: temperature with five levels, concentration with seven levels and three repetitions. In addition, the freezing point of cape gooseberry juice at various concentrations was determined. Density and viscosity values of juice decreased with increasing temperature and increased with increasing concentration of soluble solids. The effect of temperature on viscosity at different concentrations of juice was described by an Arrhenius type relationship while the activation energy of flow was found to be in the range of 7.02 to $17.62 \mathrm{~kJ} / \mathrm{mol}$. The freezing points of juice at various concentrations were determined based on the cooling curves. The results were compared with those estimated from a model applicable to fruit juices, with an average relative error of $6.6 \%$.
\end{abstract}

Keywords: cape gooseberry; density; viscosity; activation energy; freezing point 


\section{INTRODUCCIÓN}

La especie Physalis peruviana es una planta herbácea originaria de los Andes suramericanos que pertenece a la familia de las solanáceas. Los frutos de uchuva están contenidos dentro de un cáliz o capacho que los protege contra insectos, aves, patógenos y condiciones climáticas; son de color amarillo, con un diámetro entre 1.25 y $2.50 \mathrm{~cm}$, masa entre 4 y $10 \mathrm{~g}$ y se caracterizan por presentar elevados contenidos de vitaminas $\mathrm{A}$ y $\mathrm{C}$, además de hierro y fosforo. Aunque la especie Physalis peruviana crece como planta silvestre en las zonas tropicales de América, posteriormente fue introducida a África e India, existiendo hoy más de 80 ecotipos a nivel mundial (Fischer, 1995; Fischer et al., 2014). El cultivo de uchuva es una alternativa de producción para la economía de muchos países dadas las características nutricionales y propiedades medicinales que posee (Gastelum, 2012; Puente et al., 2011). A nivel mundial, Colombia es el mayor exportador de uchuva, debido que el ecotipo "Colombia" presenta una coloración amarilla brillante, mayor contenido de azúcares en el fruto (sabor dulce) y aroma pronunciado, características que han tenido gran aceptación en los mercados internacionales (Fischer, 1995; Galvis et al., 2005). Una planta produce alrededor de 300 bayas de uchuva mientras un cultivo tecnificado entre 20 y 33 toneladas por hectárea (Ramadan y Moersel, 2007).

Se ha reportado que la uchuva contiene $78.9 \%$ de humedad y $15 \%$ de sólidos solubles (principalmente azúcares), así como un alto nivel de fructosa (Fischer et al., 2014). Uno de los primeros estudios sobre la composición química y parámetros fisicoquímicos del jugo de uchuva estableció un rendimiento en la elaboración de jugo del $72.6 \pm 1.23 \%$ de la masa del fruto y que el jugo contenía $92.7 \pm 2.35 \%$ de humedad, $0.44 \pm 0.01 \%$ de proteína, $5.65 \pm 0.22 \%$ de carbohidratos y $0.2 \pm 0.02 \%$ de lípidos (Ramadan y Moersel, 2007). Los niveles de ácido ascórbico en el jugo de uchuva (46 mg/100 g) fueron mayores a los encontrados en frutas como pera, manzana y melocotón y comparables con los de naranja (50 mg/100 g) (Ramadan y Moersel, 2007; Belitz et al., 2009). El jugo de uchuva es considerado una fuente rica en ácidos grasos poliinsaturados, debido a los altos niveles de ácidos linoleico, oleico, palmítico, $\gamma$-linolénico y palmitoleico, contenidos en la fracción lipídica. Los niveles de vitamina $E$ y $\beta$-carotenos en este jugo son igualmente elevados, lo que podría convertirlo en una nueva fuente de bebidas funcionales sin que sea necesaria su fortificación con compuestos bioactivos liposolubles (Ramadan y Moersel, 2007). Los efectos de la pasteurización sobre la actividad residual de la enzima pectinmetilesterasa y las propiedades sensoriales del jugo de uchuva también han sido estudiados (Pinchao et al., 2014).

Varios compuestos bioactivos hallados en los extractos de Physalis peruviana como withanólidos, di y triglicósicos de quercetina y kaempferol, han demostrado actividad anti-hepatotóxica (Arun y Asha, 2007), anti-inflamatoria (Wu et al., 2006) y anti-hepatoma (Wu et al., 2004). Además, se le atribuyen propiedades medicinales tales como antidiabética, antiséptica, diurética, sedante y analgésica (Ahmad et al., 1999; Ramadan y Moersel, 2003; Rodríguez y Rodríguez, 2007). Dadas las características nutricionales de la uchuva y su nivel de aceptación en los mercados internacionales de frutas exóticas, Colombia incrementó la producción y comercialización, ocupando en la actualidad el segundo lugar en las exportaciones de frutas, sólo superadas por el banano (Buitrago Nuñez y Montoya Monsalve, 2012).

En la industria alimentaria, los jugos de frutas son sometidos a diferentes operaciones como pasteurización, almacenamiento en frío, congelación y concentración, con el fin de reducir los costos en transporte e incrementar su vida útil. Así pues, el conocimiento de las propiedades físicas de los jugos de fruta y su variación con los cambios en las condiciones de proceso, son fundamentales para lograr un adecuado control del producto (Ramos y lbarz, 1998). La densidad, viscosidad y la depresión del punto de congelación de un fluido son las principales variables a considerar en procesos como la crioconcentración y, a partir de estas variables se pueden realizar cálculos para el diseño de equipos, tales como el número de Reynolds, los coeficientes de transferencia de calor, el caudal de las bombas y los requerimientos energéticos del proceso (Magerramov et al., 2007; Auleda et al., 2011a). La densidad y viscosidad de los concentrados de fruta se ven influenciadas por la concentración de sólidos solubles y la temperatura de operación (Constenla et al., 1989), mientras que el punto de congelación se ve afectado, principalmente, por la concentración de azúcares presentes (Sánchez et al., 2010). Por lo anterior, el objetivo de este trabajo fue determinar el efecto de la concentración y la temperatura sobre la densidad y viscosidad del jugo de uchuva clarificado. A partir de las curvas de enfriamiento del jugo de uchuva se determinó el punto de congelación en función de la concentración, y los resultados se compararon con los estimados mediante el modelo propuesto por Auleda et al. (2011b), con base en la composición de los azúcares presentes en el jugo de fruta.

\section{MATERIALES Y MÉTODOS}

Se muestra la preparación de la solución madre de jugo concentrado de uchuva y sus diluciones, se describen los análisis fisicoquímicos del jugo de uchuva clarificado y los métodos de medición de la densidad, viscosidad y punto de congelación, terminando con una descripción del diseño experimental. 


\section{Preparación de la solución madre de jugo concentrado de uchuva y sus diluciones}

Las uchuvas (Physalis peruviana) utilizadas como materia prima para la elaboración del jugo se adquirieron en el mercado local de la ciudad de Manizales (Colombia). Los frutos maduros se lavaron cuidadosamente y se seleccionaron aquellos de consistencia firme. El jugo de uchuva se obtuvo mediante un extractor de jugos (Oster® 3169) a partir de frutos homogéneos en color y tamaño (diámetro promedio de $1.5 \mathrm{~cm}$ ). El jugo fue clarificado por filtración (papel filtro Whatman grado 1 , retención partículas $>11 \mu \mathrm{m}$ ) y concentrado en un rotavapor (Büchi R-114 Rotary Evaporator) a $45^{\circ} \mathrm{C}$ y $72 \mathrm{~mm} \mathrm{Hg}$ de presión, hasta que la concentración de sólidos alcanzó los 50 Brix. Una vez obtenida la solución madre del jugo de uchuva, se prepararon las diluciones de estudio adicionando agua destilada hasta obtener soluciones con concentraciones en un rango de 20 a $50 \stackrel{\circ}{\circ}$ Brix, con incrementos de $5 \stackrel{\circ}{\circ}$ Brix.

\section{Análisis fisicoquímicos del jugo de uchuva clarificado}

Al jugo de uchuva clarificado se le determinó: concentración de sólidos solubles (SS) a $20{ }^{\circ} \mathrm{C}$ empleando un refractómetro de Abbe (Abbe-3L Fisher Scientific, precisión $\pm 0.25^{\circ} \mathrm{Brix}$ ); $\mathrm{pH}$ con un pH-meter digital (Schott CG842, precisión \pm 0.01 unidades) y acidez total por titulación con $\mathrm{NaOH}(0.1 \mathrm{M})$ según el método 942.15 (37.1.37) de la AOAC (2012). Los niveles de glucosa, fructuosa y sacarosa en el jugo clarificado (expresados en \% en masa) se determinaron mediante cromatografía líquida de alta resolución (HPLC), empleando un equipo Hitachi modelo Elite LabChrom (Tokio, Japón), detector de índice de refracción, columna Spheriosorb $\mathrm{NH}_{2}(25 \times 0.4 \mathrm{~cm}$ ), fase móvil acetonitrilo-agua $(75: 25)$ y caudal de $1.5 \mathrm{~mL} / \mathrm{min}$ (Auleda et al., 2011b). Todos los análisis se realizaron por triplicado.

\section{Medición de la densidad}

La densidad ( $\rho$ ) del jugo de uchuva a diferentes concentraciones se determinó con un picnómetro volumétrico según el método 945.06 de la AOAC (2012). Las mediciones se realizaron a 20,25, 30, 40 y 50 C. La temperatura de la muestra se equilibró en baño termostatado (Lauda E100, precisión $\pm 0.1 \stackrel{\circ}{\circ}$ ). Los picnómetros de $5 \mathrm{~mL}$ fueron calibrados previamente a la temperatura de cada experimento. Todas las mediciones fueron realizadas por triplicado.

\section{Medición de la viscosidad}

La viscosidad ( $\eta$ ) del jugo de uchuva a diferentes concentraciones se determinó en un viscosímetro capilar tipo Ubbelohde (Fungilab, precisión $\pm 2 \%$ ) a las mismas temperaturas que la densidad. Para el control de temperatura se utilizó un baño termostatado (Lauda E100, precisión $\pm 0.1 \stackrel{\circ}{\circ}$ ). Todos los ensayos se realizaron por triplicado. Con los valores de viscosidad a diferentes temperaturas, se calculó la energía de activación de flujo para cada concentración, utilizando la ecuación 1 tipo Arrhenius (Abdulagatov et al., 2008; Zuritz et al., 2005):

$$
\eta=\eta_{\infty} \exp \left(-E_{a} / R T\right)
$$

donde $\eta$ es la viscosidad ( $\mathrm{mPa} s$ ), $\eta_{\propto}$ es una constante que corresponde a la viscosidad a una temperatura infinita ( $\mathrm{mPa} \mathrm{s}), E_{a}$ es la energía de activación de flujo $(\mathrm{kJ} / \mathrm{mol}), \mathrm{R}$ es la constante universal de los gases $(8.3144 \mathrm{~J} / \mathrm{mol} \mathrm{K})$ y $\mathrm{T}$ es la temperatura absoluta $(\mathrm{K})$. Al ajustar los valores del logaritmo natural de la viscosidad en función del inverso de la temperatura absoluta $(1 / T)$ se obtiene una línea recta, a partir de cuya pendiente es posible calcular el valor de la energía de activación.

\section{Medición y predicción del punto de congelación}

Para determinar la temperatura de congelación (TC) del jugo de uchuva a diferentes concentraciones se utilizó un equipo similar al descrito por Rahman et al. (2002). La celda cilíndrica para contener la muestra (diámetro interno $2.7 \mathrm{~cm}$, altura $12.5 \mathrm{~cm}$ y espesor de $1 \mathrm{~mm}$ ) está conectada a un baño termostatado de refrigeración Cole Palmer (USA), que utiliza como líquido de enfriamiento una mezcla etilenglicol-agua (50:50), la cual tiene una capacidad de enfriamiento de hasta $-50{ }^{\circ} \mathrm{C}$ (Telis-Romero et al., 2015). La temperatura del equipo se mantuvo a $-30 \stackrel{\circ}{\circ}$ en todos los experimentos. La celda se cargó con $20 \mathrm{~mL}$ de muestra; la temperatura en el centro de la muestra se registró cada segundo usando una termocupla tipo K, la cual está conectada a un data logger Hydra Data Bucket de Fluke (USA).

La termocupla se calibró utilizando agua bidestilada y solución de sacarosa a 20 Brix. Para la estimación del punto de congelación se empleó la ecuación 2 propuesta por Auleda et al. (2011b) para jugos de manzana, melocotón y pera. 
$\frac{M_{S-S}-M_{S-G}}{M_{S-m}-M_{S-G}}=\frac{T_{S, B x}-T_{G, B x}}{T_{m, B x}-T_{G, B x}}$

donde Ms-s, Ms-G y Ms-m son los pesos moleculares de la sacarosa, glucosa y del jugo, $T_{\mathrm{s}, \mathrm{Bx}}$ y $\mathrm{T}_{\mathrm{G}, \mathrm{Bx}}$ son los puntos de congelación de soluciones de sacarosa y glucosa a la concentración de interés (ํBrix) y $T_{\mathrm{m}, \mathrm{Bx}}$ corresponde al valor estimado del punto de congelación del jugo en estudio a la concentración de interés. Los datos estimados por medio del modelo fueron comparados con los valores experimentales.

\section{Diseño Experimental}

Para analizar el efecto de la temperatura y la concentración de sólidos solubles sobre la viscosidad y densidad del jugo de uchuva, se realizó un diseño completamente aleatorio con arreglo factorial $5 \times 7$, correspondiente a cinco niveles de temperatura $\left(20,25,30,40\right.$ y $\left.50{ }^{\circ} \mathrm{C}\right)$, siete niveles de concentración (20 a $50{ }^{\circ}$ Brix, con intervalos de $5^{\circ}$ Brix) y tres repeticiones, para un total de 105 ensayos. Los resultados experimentales se analizaron empleando la metodología de superficie de respuesta mediante el software Design-Expert® versión 8.0 (Stat-Ease, Inc., Minneapolis, USA). La bondad de ajuste del modelo se evaluó mediante el coeficiente de determinación $\left(R^{2}\right)$, el error porcentual promedio (\% E) y la raíz cuadrada del error medio cuadrático (RMSE).

\section{RESULTADOS Y DISCUSIÓN}

Los resultados se presentan en tres sub secciones: análisis fisicoquímico del jugo de uchuva, medición de la densidad y viscosidad del jugo de uchuva, y determinación experimental y predicción del punto de congelación

\section{Análisis fisicoquímico del jugo de uchuva}

Las propiedades fisicoquímicas del jugo de uchuva clarificado se muestran en la tabla 1, así como los datos reportados (Ramadan y Moersel, 2007). La proporción de azúcares en el jugo fue de $50.33 \pm 0.05 \%$ de sacarosa, $25.96 \pm 0.21 \%$ de glucosa y $23.71 \pm 0.15 \%$ de fructosa, valores similares a los encontrados en jugos de melocotón, manzana y pera (Auleda et al., 2011b).

Tabla 1: Propiedades fisicoquímicas del jugo de uchuva clarificado

\begin{tabular}{|l|l|l|}
\hline \multicolumn{1}{|c|}{ Propiedad } & \multicolumn{1}{|c|}{ Valor obtenido } & \multicolumn{1}{c|}{$\begin{array}{c}\text { Valor reportado } \\
\text { (Ramadan y Moersel, 2007) }\end{array}$} \\
\hline $\mathrm{pH}$ & $3.01 \pm 0.01$ & $3.86 \pm 026$ \\
\hline${ }^{\circ}$ Brix & $12.03 \pm 0.25$ & $10.5 \pm 0.46$ \\
\hline Acidez & $1.96 \pm 0.19 \mathrm{~g}$ ácido cítrico $/ 100 \mathrm{~mL}$ jugo & $0.92 \pm 0.09 \mathrm{~g}$ ácido cítrico $/ 100 \mathrm{~g}$ jugo \\
\hline Azúcar total & $9.48 \pm 0.57 \mathrm{~g} / 100 \mathrm{~mL}$ jugo & $4.90 \pm 0.16 \mathrm{~g} / 100 \mathrm{~g}$ jugo \\
\hline
\end{tabular}

Existen diferencias en las propiedades físicas del jugo de uchuva analizado en este trabajo y las reportadas por Ramadan y Moersel (2007) para el jugo de uchuva cultivada en Egipto, las cuales pueden estar asociadas a los diferentes ecotipos del fruto. Por ejemplo, se ha reportado que la uchuva ecotipo "Colombia" tiene mayor contenido de azúcares, lo cual se corrobora en este estudio.

\section{Medición de la densidad y viscosidad del jugo de uchuva}

Los datos experimentales obtenidos para la densidad y viscosidad del jugo de uchuva a las diferentes concentraciones y temperaturas de estudio se presentan en las tablas 2 y 3 , respectivamente. Estos valores corresponden al promedio de tres medidas experimentales y, en todos los casos, la desviación relativa estuvo por debajo del $5 \%$.

Tabla 2: Variación de la densidad del jugo de uchuva con la temperatura y la concentración

\begin{tabular}{|c|c|c|c|c|c|}
\hline \multirow[t]{2}{*}{ SS ( ${ }^{\circ}$ Brix) } & \multicolumn{5}{|c|}{ Densidad $\left(\mathrm{kg} / \mathrm{m}^{3}\right)$} \\
\hline & $20^{\circ} \mathrm{C}$ & $25^{\circ} \mathrm{C}$ & $30^{\circ} \mathrm{C}$ & $40^{\circ} \mathrm{C}$ & $50^{\circ} \mathrm{C}$ \\
\hline 20 & 1075.5 & 1057.3 & 1051.4 & 1046.2 & 1039.7 \\
\hline 25 & 1102.5 & 1087.1 & 1080.3 & 1073.1 & 1065.5 \\
\hline 30 & 1128.5 & 1118.1 & 11106 & 1101.8 & 1093.4 \\
\hline 35 & 1151.7 & 1146.8 & 1139.3 & 1129.9 & 1121.2 \\
\hline 40 & 1170.5 & 1167.2 & 1163.4 & 1154.5 & 1146.4 \\
\hline 45 & 1184.8 & 1180.9 & 1179.2 & 1172.2 & 1166.0 \\
\hline 50 & 1187.5 & 1183.4 & 1180.9 & 1179.2 & 1176.6 \\
\hline
\end{tabular}


La densidad del jugo se vio afectada en menor proporción por la temperatura. Para el rango de 20 a 50 ${ }^{\circ}$ Brix, la densidad del jugo disminuyó entre 3.33 y $0.92 \%$ con el incremento de la temperatura de 20 a $50{ }^{\circ} \mathrm{C}$; mientras que para el rango de 20 a $50{ }^{\circ} \mathrm{C}$, la densidad del jugo aumentó entre el 10.41 y $13.17 \%$ al incrementar la concentración de 20 a $50^{\circ}$ Brix.

Tabla 3: Variación de la viscosidad del jugo de uchuva con la temperatura y la concentración

\begin{tabular}{|c|c|c|c|c|c|c|c|c|}
\hline \multirow{2}{*}{ SS (Bix) } & \multicolumn{5}{|c|}{ Viscosidad (m Pa.s) } & \multirow{2}{*}{$\begin{array}{c}\text { Ea } \\
(\mathrm{kJ} / \mathrm{mol})\end{array}$} & $\eta_{\propto}(\mathrm{m} \mathrm{Pa} \mathrm{s})$ & $\mathrm{r}^{2}$ \\
\cline { 2 - 8 } & $20^{\circ} \mathrm{C}$ & $25^{\circ} \mathrm{C}$ & $30^{\circ} \mathrm{C}$ & $40^{\circ} \mathrm{C}$ & $50^{\circ} \mathrm{C}$ & & \\
\hline 20 & 2.6565 & 2.1882 & 2.0919 & 1.6296 & 1.3270 & 17.62 & $1.878 \times 10^{-3}$ & 0.988 \\
\hline 25 & 3.5369 & 3.0435 & 2.7848 & 2.3451 & 1.9472 & 15.12 & $6.977 \times 10^{-3}$ & 0.994 \\
\hline 30 & 4.6130 & 4.0888 & 3.6663 & 3.2679 & 2.7848 & 12.72 & $2.433 \times 10^{-2}$ & 0.988 \\
\hline 35 & 5.8848 & 5.3243 & 4.7619 & 4.4062 & 3.8607 & 10.58 & $7.486 \times 10^{-2}$ & 0.975 \\
\hline 40 & 7.3522 & 6.7497 & 6.0982 & 5.7667 & 5.1939 & 8.68 & $2.032 \times 10^{-1}$ & 0.962 \\
\hline 45 & 9.0152 & 8.3653 & 7.7022 & 7.3553 & 6.8023 & 7.02 & $4.930 \times 10^{-1}$ & 0.955 \\
\hline 50 & 16.1802 & 15.4952 & 14.6313 & 12.228 & 11.1139 & 10.46 & $2.252 \times 10^{-1}$ & 0.985 \\
\hline
\end{tabular}

Con respecto a la viscosidad, ésta propiedad física se vio fuertemente afectada por la temperatura y la concentración. Por ejemplo, para el rango de 20 a $50^{\circ}$ Brix, la viscosidad del jugo disminuyó entre 50.05 y $31.31 \%$ por incremento de la temperatura de 20 a $50{ }^{\circ} \mathrm{C}$; mientras que para el rango de 20 a $50{ }^{\circ} \mathrm{C}$, la viscosidad del jugo aumentó entre el 83.58 y $88.06 \%$ al incrementar la concentración de 20 a 50 Brix. En general, para una concentración fija de sólidos solubles, la densidad y viscosidad del jugo de uchuva disminuyeron con el aumento en la temperatura. La disminución en la densidad está asociada a la expansión volumétrica que sufre la solución por efecto del incremento en la temperatura (Constenla et al., 1989), en tanto que disminución en la viscosidad se atribuye a la reestructuración espacial y al aumento de la distancia intermolecular debida al el incremento de la temperatura (Haminiuk et al., 2006). Para un valor fijo de temperatura, la densidad y viscosidad del jugo aumentaron al incrementarse la concentración de sólidos solubles. Al aumentar la concentración de sólidos solubles en el jugo, se genera una mayor interacción soluto-agua y restricción en el movimiento molecular, por ende, la viscosidad del jugo se incrementa (Constenla et al., 1989). Comportamientos similares en las propiedades termofísicas de jugos de frutas han sido observados en otros estudios (Ramos y lbarz, 1998; Magerramov et al., 2007).

En la tabla 3 se muestran los valores de energía de activación del flujo ( $\left.E_{a}\right)$ y la constante $\eta_{\infty}$ (mPa s) obtenidos al ajustar el comportamiento de la viscosidad al modelo tipo Arrhenius. Para el rango de 20 a 45 'Brix, la $\mathrm{E}_{\mathrm{a}}$ disminuyó linealmente con el incremento en la concentración del jugo de uchuva, comportamiento similar al observado en el jugo de mango (Dak et al., 2006). Para 50 Brix, la energía de activación no conservó la tendencia anterior, sugiriendo que a esta concentración las propiedades reológicas del jugo cambian considerablemente o que el modelo de Arrhenius para la viscosidad no es aplicable. La ecuación (1) presenta fallas al representar los datos experimentales de viscosidad de jugos de frutas a altas temperaturas y elevada concentración de sólidos (Abdulagatov et al., 2008).

El valor más alto de $E_{a}$ se obtuvo a $20^{\circ}$ Brix, lo cual indica que para el jugo en estudio el efecto de la temperatura sobre la viscosidad es mayor a concentraciones bajas. Los valores de $\mathrm{E}_{\mathrm{a}}$ obtenidos para el jugo de uchuva se encuentran dentro del rango reportado para jugos de mango ( $E_{a}$ entre 1.66 y 11.35 $\mathrm{kJ} / \mathrm{mol}$ )(Dak et al., 2006) y uva clarificado ( $E_{\mathrm{a}}$ entre 16.33 y $52.02 \mathrm{~kJ} / \mathrm{mol}$ ) (Zuritz et al., 2005). Normalmente, en los jugos de fruta que tienen un comportamiento newtoniano, la energía de activación de flujo aumenta con la concentración de sólidos (Abdulagatov et al., 2008; Zuritz et al, 2005). Sin embargo, en el jugo de mango, la energía de activación de flujo disminuye al aumentar la concentración, debido a la naturaleza seudoplástica de este fluido (Dak et al., 2006).

Al realizar el ajuste de los datos experimentales de densidad y viscosidad, empleando la metodología de superficie de respuesta, se obtuvo una ecuación polinómica no lineal de segundo orden, como una función de la temperatura (T) y la concentración de sólidos solubles (SS). Los modelos obtenidos, el coeficiente de determinación $\left(R^{2}\right)$, el error porcentual promedio (\%E) y la raíz cuadrada del error medio cuadrático (RMSE) se presentan en la tabla 4 . Se considera que para un $R^{2}>0.85$, \%E $<10 \%$ y valores pequeños de RMSE $(<0.3)$ (Cerón et al., 2016), los valores pronosticados por el modelo son muy cercanos a los valores reales. El modelo de densidad cumple con todos los criterios de ajuste, sin embargo, la viscosidad presenta un \%E mayor al recomendado. El error porcentual promedio superior al 10\% se asocia al incremento excesivo de la viscosidad a $50{ }^{\circ}$ Brix en el rango de temperatura estudiado, el cual se refleja en la variación de la tendencia en la energía de activación de flujo. 
Tabla 4: Modelos de ajuste para la densidad y viscosidad del jugo de uchuva

\begin{tabular}{|c|l|c|c|c|}
\hline \multirow{2}{*}{ Variable } & \multicolumn{1}{|c|}{ Ecuación del modelo } & \multicolumn{1}{|c|}{ Criterios de ajuste } \\
\cline { 2 - 5 } & \multicolumn{1}{|c|}{$\mathrm{R}^{2}$} & $\% \mathrm{E}$ & $\mathrm{RMSE}$ \\
\hline Densidad & $\begin{array}{l}\rho=949.897-2.959 \times T+9.851 \times S S+1.778 \times 10^{-2} \times T^{2}+ \\
2.411 \times 10^{-2} \times \mathrm{T} \times S S-8.860 \times 10^{-2} \times S S^{2}\end{array}$ & 0.994 & 2.805 & 0.003 \\
\hline Viscosidad & $\begin{array}{l}\eta=10.1547-0.0503570 \times T-0.558108 \times S S- \\
0.00327053 \times T \times S S+0.0144775 \times S S^{2}\end{array}$ & 0.952 & 14.038 & 0.179 \\
\hline
\end{tabular}

La figura 1 muestra los gráficos tridimensionales de las superficies de respuesta obtenidas para la densidad (A) y viscosidad (B) del jugo de uchuva en función de la temperatura y la concentración de sólidos solubles, según las ecuaciones del modelo mostradas en la tabla 4. Los resultados obtenidos del análisis de varianza a un nivel de confianza del $95 \%$ indican que la temperatura (T), la concentración del jugo (SS) y su interacción (TxSS), tienen un efecto estadísticamente significativo sobre la densidad y viscosidad, reflejándose en los coeficientes de los modelos. Los coeficientes cuadráticos de temperatura $\left(\mathrm{T}^{2}\right)$ y la concentración del jugo $\left(\mathrm{SS}^{2}\right)$ presentan un efecto significativo en la densidad del jugo, en tanto que para la viscosidad sólo el coeficiente SS $^{2}$ es relevante en el modelo.

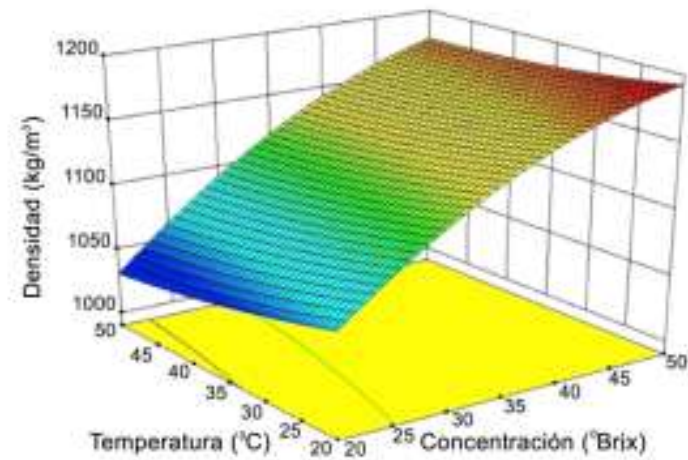

(A)

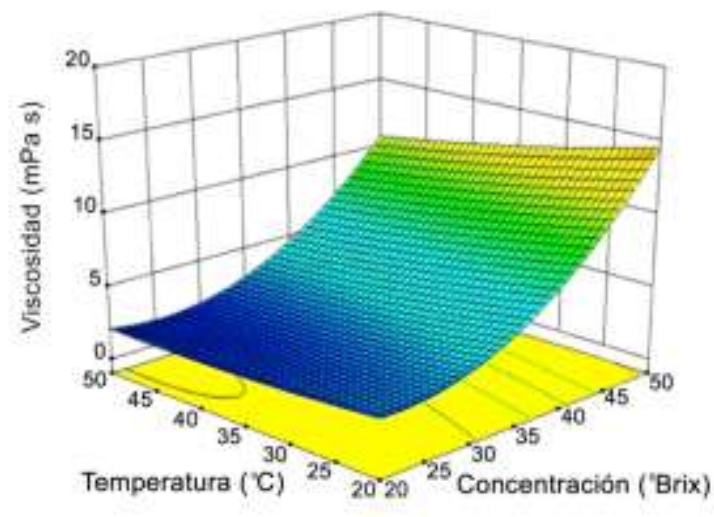

(B)

Fig 1: Variación de la densidad (A) y viscosidad (B) del jugo de uchuva con la temperatura y la concentración de sólidos solubles

A partir de los modelos cuadráticos obtenidos con los datos experimentales se calcularon las condiciones óptimas, encontrándose una densidad máxima de $1190.0 \mathrm{Kg} / \mathrm{m}^{3}$ a $61^{\circ}$ Brix y $41^{\circ} \mathrm{C}$, y una viscosidad mínima de $1.8178 \mathrm{mPa}$.s a $25{ }^{\circ}$ Brix y $52{ }^{\circ} \mathrm{C}$. Para la concentración de $62{ }^{\circ}$ Brix no se realizaron ensayos experimentales, debido a que el jugo se uchuva tiene una viscosidad muy alta y presenta un comportamiento seudoplástico.

\section{Determinación experimental y predicción del punto de congelación}

Los puntos de congelación del jugo de uchuva a varias concentraciones fueron determinados experimentalmente a partir de las curvas de enfriamiento de cada una de las soluciones. Con base en la concentración relativa de los azúcares presentes en el jugo, se calculó el peso molecular efectivo del jugo de uchuva, aplicando la ecuación 3 propuesta por Chen et al. (1986):

$\mathrm{M}_{\mathrm{S}-\mathrm{m}}=\frac{\% \mathrm{~F} * \mathrm{mF}}{100}+\frac{\% \mathrm{G} * \mathrm{mG}}{100}+\frac{\% \mathrm{~S} * \mathrm{mS}}{100}$

donde \%F, \%G y \%S representan los porcentajes de fructosa, glucosa y sacarosa; $\mathrm{mF}, \mathrm{mG}$ y $\mathrm{mS}$ son los pesos moleculares de la fructosa, glucosa y sacarosa; y Ms-m es el peso molecular para el jugo en estudio, cuyo valor obtenido fue de $262.49 \mathrm{~g} / \mathrm{mol}$. A partir de este valor y los puntos de congelación de las soluciones de sacarosa y glucosa reportados por Auleda et al. (2011b), se calcularon los puntos de congelación del jugo de uchuva a diferentes concentraciones. La figura 2 muestra la tendencia de la temperatura de congelación del jugo de uchuva en función de la concentración. En la gráfica se verifica que la curva obtenida para este jugo está acotada por la envolvente superior de la sacarosa y la inferior de la glucosa, tal como lo establecieron Auleda et al. (2011b) para los jugos de melocotón, manzana y pera. 


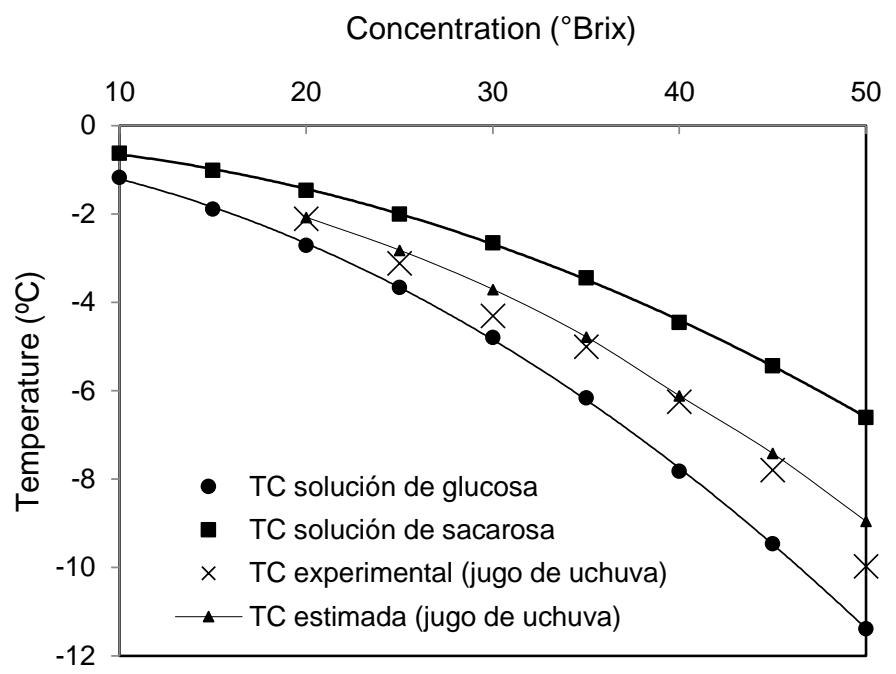

Fig 2: Temperatura de congelación (TC) experimental y estimada para el jugo de uchuva comparada con las curvas de TC de las soluciones de sacarosa (superior) y glucosa (inferior)

Al incrementar la concentración de sólidos solubles en el jugo de uchuva se obtiene un descenso en la temperatura de congelación de $-2.10^{\circ} \mathrm{C}\left(20^{\circ}\right.$ Brix $),-3.11^{\circ} \mathrm{C}\left(25^{\circ} \mathrm{Brix}\right),-4.30^{\circ} \mathrm{C}\left(30^{\circ} \mathrm{Brix}\right),-5.00^{\circ} \mathrm{C}(35$ ${ }^{\circ}$ Brix $),-6.24^{\circ} \mathrm{C}\left(40^{\circ} \mathrm{Brix}\right),-7.79^{\circ} \mathrm{C}\left(45^{\circ} \mathrm{Brix}\right)$ y $-9.97^{\circ} \mathrm{C}\left(50^{\circ} \mathrm{Brix}\right)$. Las temperaturas de congelación del jugo de uchuva estimadas a partir de la correlación establecida por Auleda et al. (2011b) para jugos de frutas, son ligeramente menores que las obtenidas experimentalmente, con un error relativo promedio del $6.60 \%$.

\section{CONCLUSIONES}

De los resultados mostrados se pueden obtener las siguientes conclusiones sobre el efecto de la concentración y la temperatura en las propiedades físicas del jugo de uchuva clarificado: 1) la densidad y viscosidad del jugo de uchuva se pueden correlacionar con la concentración de sólidos solubles y la temperatura, mediante una ecuación polinómica no lineal de segundo orden; 2) el análisis de varianza, a un nivel de confianza del $95 \%$, indica que tanto la temperatura como la concentración de sólidos solubles en el jugo de uchuva tienen un efecto estadísticamente significativo sobre los valores de densidad y viscosidad; 3) el efecto de la temperatura sobre la viscosidad del jugo de uchuva a diferentes concentraciones puede ser descrito por una relación tipo Arrhenius; 4) la variación de la energía de activación de flujo muestra que el efecto de la temperatura sobre la viscosidad es mayor a concentraciones bajas y 5) la estimación del punto de congelación del jugo de uchuva, con base en las concentraciones relativas de los azúcares presentes en el jugo, se ajustó muy bien a los valores experimentales obtenidos.

\section{AGRADECIMIENTOS}

Los autores agradecen a la Dirección de Investigaciones de la Universidad Nacional de Colombia, Sede Manizales (DIMA) por la financiación del proyecto.

\section{REFERENCIAS}

Abdulagatov, A.I.; M.A. Magerramov, I.M. Abdulagatov y N.D. Azizov, Chapter 2. Effect of temperature, pressure and concentration on the viscosity of fruit juices: Experimental and Modeling, Cantor, J.M. (Ed), pp. 61-130, Nova Science Publisher Inc., New York, USA (2008)

Ahmad, S.; A. Malik; R. Yasmin; N. Ullah; W. Gul; P.M. Khan; H.R. Nawaz y N. Afza, Withanolides from Physalis peruviana, doi:10.1016/S0031-9422(98)00567-6, Phytochemistry: 50(4), 647-651 (1999)

AOAC. Official Methods of Analysis of AOAC International, 18th. Ed. AOAC International, GaithersburgMaryland, USA (2012)

Arun, M. y V.V. Asha, Preliminary studies on antihepatotoxic effect of Physalis peruviana Linn. (Solanaceae) against carbon tetrachloride induced acute liver injury in rats, doi:10.1016/j.jep.2006.10.038, J Ethnopharmacol, 111(1), 110-114 (2007) 
Auleda, J.M.; M. Raventós y E. Hernández, Calculation method for designing a multi-plate freezeconcentrator for concentration of fruit juices, doi:10.1016/j.jfoodeng.2011.06.006, J Food Eng, 107(1), 27-35 (2011a)

Auleda, J.M.; M. Raventós; J. Sánchez y E. Hernández, Estimation of the freezing point of concentrated fruit juices for application in freeze concentration, doi:10.1016/j.jfoodeng.2011.02.035, J. Food Eng, 105(2), 289294 (2011b)

Belitz, H.D.; W. Grosch y P. Schieberle, Fruits and fruit products, in: Food Chemistry, 4th Ed., pp. 807-861, Berlin, Germany (2009)

Buitrago-Nuñez, D.A. y J.N. Montoya-Monsalve, Modelo de costeo para la producción y comercialización de uchuva, http://revistas.ustatunja.edu.co/index.php/ivestigium/article/viewFile/365/639, ISSN: 2011-9836, In Vestigium Ire, 5(1), 47-58 (2012)

Cerón, A.F.; O. Osorio y L.F. Garcés, Procesamiento de arvejas (Pisum sativum L.). Parte 2: Cinética de adsorción de agua en semillas de arveja, variedad San Isidro y Sureña, doi:10.4067/S071807642016000100010, Inf Tecnol, 27(1), 81-90 (2016)

Constenla, D.T.; J.E. Lozano y G.H. Crapiste, Thermophysical Properties of Clarified Apple Juice as a Function of Concentration and Temperature, doi: 10.1111/j.1365-2621.1989.tb04677.x, J Food Sci, 54(3), 663-668 (1989)

Chen, C.S., Effective molecular weight of aqueous solutions and liquid foods calculated from the freezing point depression, doi:10.1111/j.1365-2621.1986.tb13853.x, J Food Sci, 51(6), 1537-1539 (1986)

Dak, M.; R.C. Verma y G.P. Sharma, Flow characteristics of juice of "Totapuri" mangoes, doi:10.1016/j.jfoodeng.2005.06.002, J Food Eng, 76(4), 557-561 (2006)

Fischer, G. Effect of root zone temperature and tropical altitude on the growth, fruit quality and development of the cape gooseberry (Physalis peruviana L.), Ph.D. Thesis, Faculty of Agriculture and Horticulture, Humboldt Universität zu Berlin, Berlin (1995)

Fischer, G.; P.J. Almanza-Merchán y D. Miranda, Importancia y cultivo de la uchuva (Physalis peruviana L.), doi: 10.1590/0100-2945-441/13, Rev Bras Frutic, 36(1), 01-15 (2014)

Galvis, J.A.; G. Fischer y O.P. Gordillo, Cosecha y poscosecha de la uchuva, in: Avances en cultivo, poscosecha y exportación de la uchuva (Physalis peruviana L.) en Colombia, Fischer, G, Miranda, D., Piedrahita, W., Romero, J. (Eds), Unibiblos-Universidad Nacional de Colombia, pp. 165-190, Bogotá, Colombia (2005)

Gastelum, D. Demanda nutrimental y manejo agronómico del cultivo Physalis peruviana L., Tesis de Magíster, Instituto de Enseñanza e Investigación en Ciencias Agrícola, Colegio de Posgraduados, Texcoco, México (2012)

Haminiuk, C.W.I; M.R. Sierakowski, J.R.M.B Vidal y M.L. Masson, Influence of temperature on the rheological behavior of whole araçá pulp (Psidium cattleianum sabine), doi:10.1016/j.Iwt.2005.02.011, LWT Food Sci Technol, 39(4), 427-431 (2006)

Magerramov, M.A.; A.I. Abdulagatov; N.D. Azizov y I.M. Abdulagatov, Effect of temperature, concentration, and pressure on the viscosity of pomegranate and pear juice concentrates, doi:10.1016/j.jfoodeng.2006.05.030, J Food Eng, 80(2), 476-489 (2007)

Pinchao, Y.A.; O. Osorio y D. Mejía, Inactivación Térmica de Pectinmetilesterasa en Jugo de Uchuva (Physalis peruviana L.), doi:10.4067/S0718-07642014000500009, Inf Tecnol, 25(5), 55-64 (2014)

Puente, L.A.; C.A. Pinto-Muñoz; E.S. Castro y M. Cortés, Physalis peruviana Linnaeus, the multiple properties of a highly functional fruit: A review, doi:10.1016/j.foodres.2010.09.034, Food Res Int, 44(7), 1733-1740 (2011)

Rahman, M.S.; N. Guizani; M. Al-Khaseibi; S. Ali Al-Hinai; S.S. Al-Maskri y K. Al-Hamhami, Analysis of cooling curve to determine the end point of freezing, doi:10.1016/S0268-005X(02)00031-0, Food Hydrocolloid, 16(6), 653-659 (2002) 
Ramadan, M.F. y J.T. Moersel, Oil goldenberry (Physalis peruviana L.), doi:10.1021/jf020778z, J Agr Food Chem, 51(4), 969-974 (2003)

Ramadan, M.F. y J.T. Moersel, Impact of enzymatic treatment on chemical composition, physicochemical properties and radical scavenging activity of goldenberry (Physalis peruviana L.) juice, doi:10.1002/jsfa.2728, J Sci Food Agric, 87(3), 452-460 (2007)

Ramos, A.M. y A. Ibarz, Density of juice and fruit puree as a function of soluble solids content and temperature, doi:10.1016/S0260-8774(98)00004-1, J Food Eng, 35(1), 57-63 (1998)

Rodríguez, S. y E. Rodríguez, Efecto de la ingesta de Physalis peruviana (aguaymanto) sobre la glicemia postprandial en adultos jóvenes, http://sisbib.unmsm.edu.pe/bvrevistas/rmv/v04n1/pdf/a05v4n1.pdf, ISSN: 1817-2075, Rev Med Vallejiana, 4(1), 43-52 (2007)

Sánchez, J.; Y. Ruiz; M. Raventós; J.M. Auleda y E. Hernández, Progressive freeze concentration of orange juice in a pilot plant falling film, doi:10.1016/j.ifset.2010.06.006, Innov Food Sci \& Emerg Technol, 11(4), 644$651(2010)$

Telis-Romero, J.; G.I. Giraldo-Gómez; H.A. Villa-Vélez; D.M. Cano-Higuita y V.R.N. Telis, Chapter 33. Influence of fluid concentration on freezing-point depression and thermal conductivity of frozen Physalis juice, in: Water Stress in Biological, Chemical, Pharmaceutical and Food Systems, pp. 393-403, doi: 10.1007/978-1-4939-2578-0_33, Springer, New York, USA (2015)

Wu, S.-J.; L.-T. Ng; C.-H. Chen; D.-L. Lin; S.-S. Wang y C.-C. Lin, Antihepatoma activity of Physalis angulata and P. peruviana extracts and their effects on apoptosis in human Hep G2 cells, doi:10.1016/j.lfs.2003.09.058, Life Sci, 74(16), 2061-2073 (2004)

Wu, S.J.; J.Y. Tsai; S.P. Chang; D.L. Lin; S.S. Wang; S.N. Huang y L.T. Ng, Supercritical carbon dioxide extract exhibits enhanced antioxidant and anti-inflammatory activities of Physalis peruviana, doi:10.1016/j.jep.2006.05.027, J Ethnopharmacol, 108(3), 407-413 (2006)

Zuritz, C.A.; E.M. Puntes; H.H. Mathey; E.H. Pérez; A. Gascón; L.A. Rubio; C.A. Carullo; R.E. Chernikoff y M.S. Cabeza, Density, viscosity and coefficient of thermal expansion of clear grape juice at different soluble solid concentrations and temperatures, doi:10.1016/j.jfoodeng.2004.10.026, J Food Eng, 71(2), 143-149 (2005). 
